\title{
Genomic Overlap between Platelet Parameters Variability and Age at Onset of Parkinson Disease
}

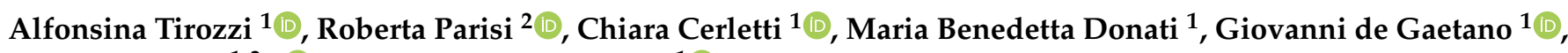 \\ Licia Iacoviello ${ }^{1,3, * \mathbb{B}}$ and Alessandro Gialluisi ${ }^{1}$ \\ 1 Department of Epidemiology and Prevention, IRCCS NEUROMED, 86077 Pozzilli, Italy; \\ nina.tirozzi@gmail.com (A.T.); chiara.cerletti@moli-sani.org (C.C.); mbdonati@moli-sani.org (M.B.D.); \\ giovanni.degaetano@moli-sani.org (G.d.G.); alessandro.gialluisi@gmail.com (A.G.) \\ 2 Department of Medicine and Health Sciences "Vincenzo Tiberio", University of Molise, \\ 86100 Campobasso, Italy; roberta.parisi@moli-sani.org \\ 3 Department of Medicine and Surgery, University of Insubria, 21100 Varese, Italy \\ * Correspondence: licia.iacoviello@moli-sani.org; Tel.: +39-0865-915247; Fax: +39-0865-927575
}

check for updates

Citation: Tirozzi, A.; Parisi, R.; Cerletti, C.; Donati, M.B.; de Gaetano, G.; Iacoviello, L.; Gialluisi, A. Genomic Overlap between Platelet Parameters Variability and Age at Onset of Parkinson Disease. Appl. Sci. 2021, 11, 6927. https://doi.org/10.3390/ app11156927

Academic Editor: Francisco Arrebola

Received: 1 April 2021

Accepted: 21 July 2021

Published: 28 July 2021

Publisher's Note: MDPI stays neutral with regard to jurisdictional claims in published maps and institutional affiliations.

Copyright: (c) 2021 by the authors. Licensee MDPI, Basel, Switzerland. This article is an open access article distributed under the terms and conditions of the Creative Commons Attribution (CC BY) license (https:// creativecommons.org/licenses/by/ $4.0 /)$.

\begin{abstract}
With the increasing burden of common neurodegenerative disorders and their longhypothesized link with platelet biology, genomic approaches have been recently used to investigate the presence of a shared genetic basis between neurodegenerative risk and platelet parameters, reporting a significant though moderate genetic correlation between Parkinson Disease (PD) risk and platelet distribution width, an index of platelet size variability. Here, we investigated the genetic overlap of platelet parameters with an endophenotype of PD, age-at-onset (PD-AAO). First, we applied a Linkage Disequilibrium (LD)-score regression to the summary statistics of a large independent Genome Wide Association Study (GWAS) previously conducted, to estimate the co-heritability based on common genetic variants. Then, we analyzed multitrait single-variant associations to identify novel loci associated with both PD-AAO and mean platelet volume (MPV). Finally, we performed gene and gene-set enrichment analyses of these associations. We observed a statistically significant genetic correlation between MPV and PD-AAO (rg (SE) $=-0.215(0.082) ; p=0.009)$. The multitrait analysis revealed eight novel variants associated with PD-AAO and 33 with MPV. The genes most significantly enriched for associations with PD-AAO included ARHGEF3 (Rho Guanine Nucleotide Exchange Factor 3), previously associated with depression, and KALRN (Kalirin RhoGEF Kinase), encoding a PINK1 interactor previously implicated in schizophrenia, Alzheimer Disease and PD itself. Interestingly, these genes were also identified in the analysis of MPV. The most significant gene-set enrichments shared between MPV and PD-AAO were observed for coagulation- and megakaryopoiesis-related pathways. These findings provide novel hints into the common genetic basis of PD endophenotypes, platelet biology and its neuropsychiatric comorbidities, paving the way for investigating common underlying mechanisms.
\end{abstract}

Keywords: neurodegenerative disorders; Parkinson disease; blood platelets parameters; genetic correlation; age at onset; systemic inflammation; KALRN; ARHGEF3

\section{Introduction}

Neurodegenerative disorders like Parkinson Disease (PD) suffer from a lack of easy-tomeasure biomarkers which can predict their incident risk before the onset of symptoms [1]. With this regard, platelets have received increasing attention in the last few years [2]. As for PD, few studies have investigated the association between the disease status and/or severity and platelet parameters like platelet count (Plt), mean platelet volume (MPV) and platelet distribution width (PDW), reporting partly consistent evidence. An epidemiological study reported an increased MPV in PD cases vs. controls [3], while another paper identified no significant differences of MPV (and Plt) [4]. However, both studies reported a 
consistently negative correlation between MPV and a PD staging scale, the Hoehn \& Yahr score $[3,4]$.

More recently, genomic approaches have been used to dissect the relationship between platelet parameters and PD risk. These methods are based on the use of summary statistics from large Genome Wide Association Studies (GWAS), which analyze millions of common genetic variants like Single Nucleotide Polymorphisms (SNPs) and small insertions/deletions (indels) throughout the genome, testing them for associations with traits and/or disorders. In a large GWAS of PD risk (56,300 PD cases and $\sim 1.4$ million controls), the authors detected no significant genetic correlations with Plt and MPV [5], tested through Linkage Disequilibrium (LD) Score Regression [6,7]. This measure, representing the SNP-based co-heritability between two traits/disorders, recently allowed our group to observe a moderate but significant genomic overlap between PDW and PD risk [1]. Here, we follow up this study by testing the co-heritability between platelet parameters, as tested before [8], and the age at onset (PD-AAO), a common endophenotype of PD [9]. While a link between the former and the latter has never been tested at the epidemiological level, we believe that genomic investigations may provide hints into the underlying genetics and biology of these traits. For this reason, we also applied a multitrait analysis of GWAS to further disentangle the genetic correlation detected between MPV and PD-AAO at the single-variant, gene and pathway level. Overall, this work is aimed at clarifying the shared genetic basis of platelet biology and peculiar PD aspects.

\section{Subjects and Methods}

All the analyses presented below are based on publicly available GWAS summary statistics (see Data Availability section).

\subsection{Linkage Disequilibrium Score Regression}

We applied an LD-score regression [6,7] to a large independent GWAS previously conducted on PD age at onset (PD-AAO) [9] and platelet parameters, namely Plt, MPV and PDW $\left(\mathrm{N}_{\max }=166,066\right)$ [8] (see Table 1). LD score regression models the genetic correlation between two traits as a function of the LD score among SNPs in 1 cM bins genome-wide, through the formula:

$$
\mathrm{r}_{\mathrm{g}}=\rho_{\mathrm{g}} / \sqrt{ }_{\mathrm{h}_{1}}{ }^{2 *} \mathrm{~h}_{2}{ }^{2}
$$

where $\rho_{\mathrm{g}}$ is the genetic covariance between trait 1 and trait 2 , and $\mathrm{h}_{1}{ }^{2}$ and $\mathrm{h}_{2}{ }^{2}$ represent the SNP-based heritability of the two traits [6,7]. The SNP-based heritability is in turn computed as the slope of the linear function between the $\chi^{2}$ association statistics and LD score (i.e., the sum of $\mathrm{r}^{2}$ of a given SNP with all the other SNPs in a $1 \mathrm{cM}$ window), for every SNP tested genome-wide (i.e., for which the association statistics are available in a given GWAS study). For this analysis, we only retained common SNPs (Minor Allele Frequency $>5 \%$ ) in the HapMap 3 EUR reference panel [10] — excluding the HLA region-since they have good imputation quality stats $\left(\mathrm{r}^{2}>0.9\right)$ in most studies [7]. The LD scores of these variants were derived using the $1000 \mathrm{G}$ phase 1 v3 EUR panel [11]. Similarly, from each study we filtered out variants that were not SNPs (i.e., indels), strand-ambiguous SNPs, and SNPs with duplicated rs numbers or with MAF $\leq 1 \%$. After quality control (QC), 904,009, 903,998 and 903,772 variants remained for the Plt, MPV and PDW GWAS respectively, with both the LD scores and summary statistics for PD-AAO available (discordant alleles removed, Table 1).

Table 1. Genetic correlations of platelet parameters with Parkinson-AAO.

\begin{tabular}{cccccc}
\hline Platelet Parameter & Variants before/after QC & rg & se & $\mathbf{z}$ & $p$ \\
\hline Plt & $1,180,459 / 904,009$ & 0.149 & 0.075 & 1.99 & 0.047 \\
\hline MPV & $\mathbf{1 , 1 8 0 , 4 3 7 / 9 0 3 , 9 9 8}$ & $-\mathbf{0 . 2 1 5}$ & $\mathbf{0 . 0 8 2}$ & $\mathbf{- 2 . 6 1}$ & $\mathbf{0 . 0 0 9}$ \\
\hline PDW & $1,180,156 / 903,772$ & -0.126 & 0.087 & -1.45 & 0.146 \\
\hline
\end{tabular}


Table 1. Genetic correlations of platelet parameters with PD-AAO, based on LD score regression analyses. Significant genetic correlations surviving the Bonferroni correction $(p<0.017)$ are highlighted in bold. Legend: Plt $=$ platelet count; MPV = mean platelet volume; PDW = platelet distribution width; $\mathrm{rg}(\mathrm{SE})=$ genetic correlation and relevant Standard Error.

\subsection{Multitrait Genetic Association Analysis}

The genetic relationship between PD-AAO and MPV was deepened through a multitrait analysis of GWAS (MTAG) [12], a method representing a generalization of the inverse-variance-weighted meta-analysis between two genetically correlated traits, which exploits LD score regression to account for (possibly unknown) sample overlap between the source GWAS and generates trait-specific effect estimates for each SNP ("conditional" on the correlated trait). MTAG is designed to improve statistical power to identify trait-specific genetic associations and novel associations in any one of the traits [13]. For our MTAG analysis, we selected the SNPs with MAF $\geq 1 \%$ that were present both in PD-AAO and MPV GWAS summary data. The canonical genome-wide significance threshold was used for this analysis $\left(\alpha=5 \times 10^{-8}\right)$.

To gain further biological insights, single-variant multitrait associations with both PDAAO ("conditioned" on MPV) and MPV ("conditioned" on PD-AAO) underwent gene and pathway enrichment tests through MAGMA v1.08 [14], within the FUMA platform [15]. In this case, a Bonferroni correction for multiple testing was adapted to the number of genes with annotated variants (within a $10 \mathrm{~kb}$ upstream/downstream interval; 18,926) and to the number of pathways with annotated genes $(15,481)$, resulting in corrected $\alpha$ thresholds of $2.6 \times 10^{-6}$ and $3.2 \times 10^{-6}$, respectively.

\section{Results}

LD-score regression revealed significant genetic correlations of PD-AAO with both Plt (rg (SE) $=0.15(0.07) ; p=0.047)$ and MPV (rg (SE) $=-0.21(0.08) ; p=0.009)$; the former, however, did not survive the correction for multiple testing for three different platelet parameters $(\alpha=0.017$; Table 1$)$.

When we followed up the significant genetic correlation between MPV and PD-AAO through multitrait association testing, we observed 5409 genome-wide significant singlevariant associations $\left(p<5 \times 10^{-8}\right)$ with $\mathrm{PD}-\mathrm{AAO}$ and 2438 significant associations with MPV (Figure 1a,b). Of these, eight were novel with PD-AAO and 33 with MPV (Table 2), compared to the original GWAS [16]. The most significant association with PD-AAO was detected at rs73186248 $\left(\beta(\mathrm{SE})=-0.933(0.16) ; p=5.57 \times 10^{-9}\right)$, while the most significant association with MPV was observed at rs17477708 ( $\beta$ (SE) $=-0.038(0.007)$; $\left.p=3.26 \times 10^{-8}\right)$.

The dashed line represents the genome-wide significant association threshold $\left(\alpha=5 \times 10^{-8}\right)$.

The analysis of 18,926 genes with annotated variants revealed 971 genes with significant enrichments of associations surviving the Bonferroni correction $\left(p<2.6 \times 10^{-6}\right)$. Of these, 173 were detected with PD-AAO (Figure 2a; Table S1a) and 798 with MPV (Figure 2b; Table S1b). The most enriched genes with PD-AAO were ARHGEF3 (Rho Guanine Nucleotide Exchange Factor 3; 3p14.3), KALRN (Kalirin RhoGEF Kinase; 3q21.1-2) and IQGAP2 (IQ motif containing GTPase activating protein 2; 5q13.3) (Figure 2a; Table S1a). These genes were also included among the most enriched genes for associations with MPV (Figure 2b; Table S1b). Gene-set enrichment tests revealed 16 pathways with significant enrichments of gene-based associations with PD-AAO (Table 3a; Table S1c) and 13 with MPV (Table 3b; Table S1d). Again, an overlap was found for associated pathways, e.g., for the Gene Ontology (GO) terms coagulation, megakaryocyte development and platelet production, and for the Curated gene sets reactome hemostasis and reactome erythropoietin activates phospholipase c gamma plcg. 
(a)

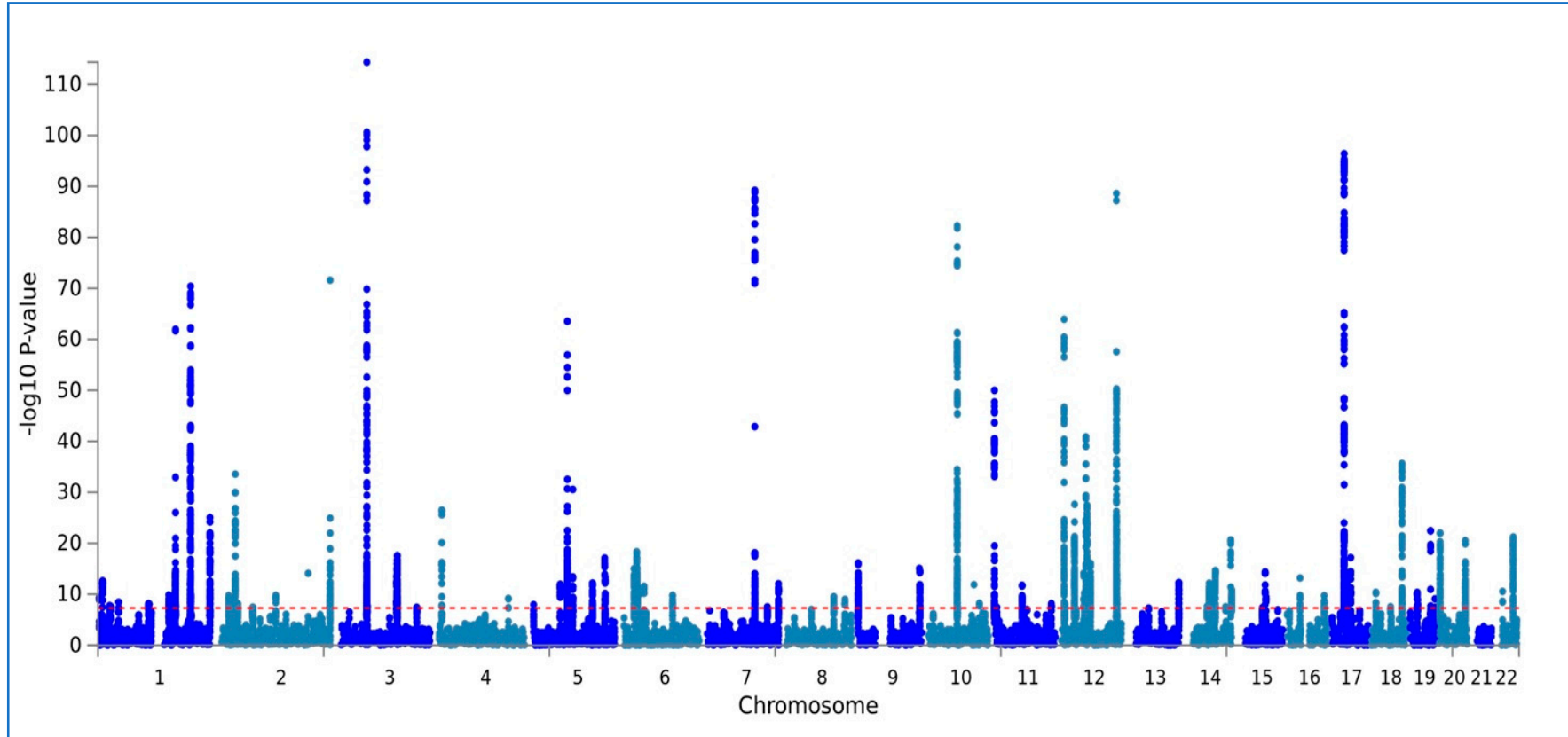

(b)

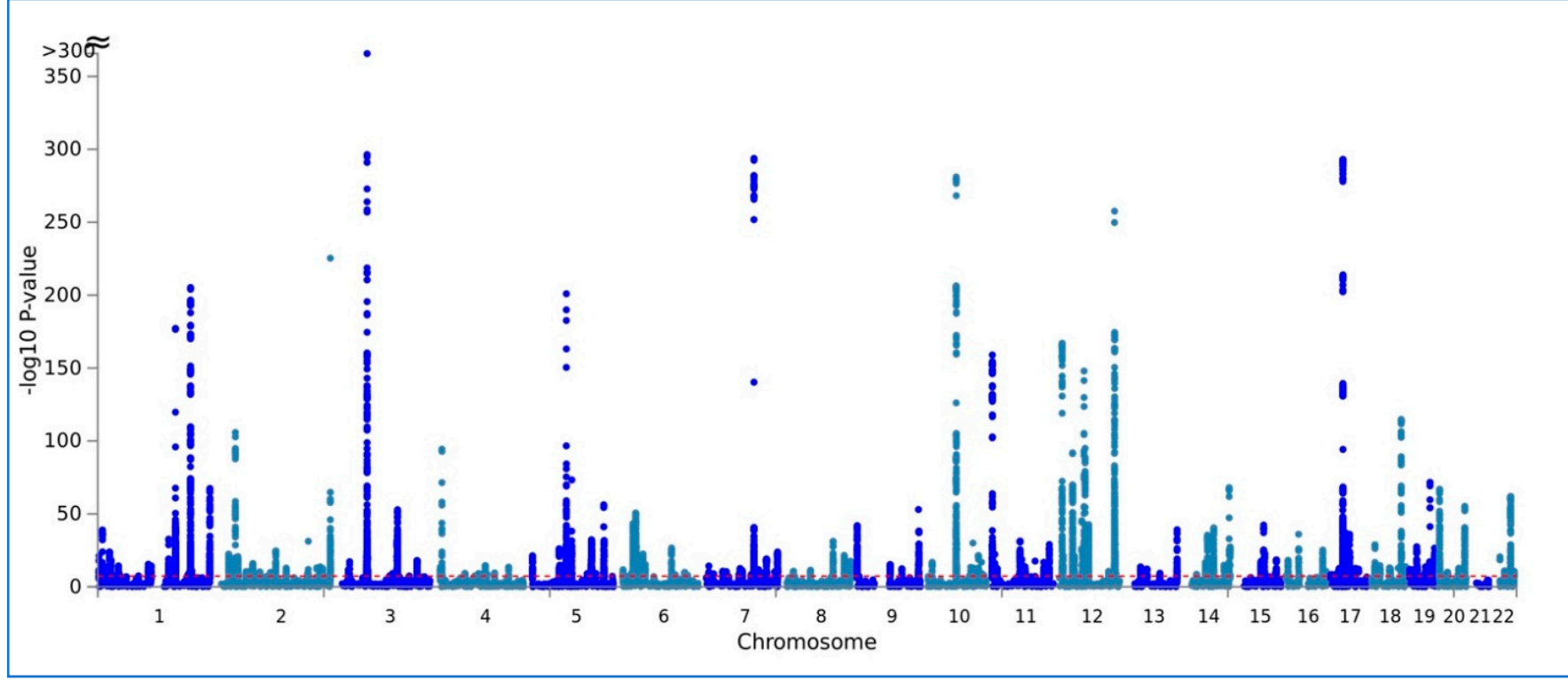

Figure 1. Manhattan Plot of multitrait single-variant associations with (a) PD-AAO and (b) MPV. 
Table 2. Novel variants associated with (a) PD-AAO and (b) MPV in a multitrait association test with MTAG.

\begin{tabular}{|c|c|c|c|c|c|c|c|c|c|c|c|c|c|}
\hline (a) & & & & & & & & & & & & & \\
\hline SNP & CHR:BP & A1 & A2 & Consequence & IMPACT & Gene & A1freq & $\begin{array}{c}\text { Beta } \\
\text { (SE)-MTAG }\end{array}$ & p-MTAG & $\begin{array}{c}\text { Beta } \\
\text { (SE)-PD_AAO }\end{array}$ & P-PD_AAO & Beta(SE)-MPV & P-MPV \\
\hline rs73186248 & 7:106423109 & A & G & upstream_gene_variant & M & RP5-884M6.1 & 0.09 & $-0.93(0.16)$ & $5.57 \times 10^{-9}$ & $-1084(0.258)$ & 0.00001781 & $-0.037(0.007)$ & $7.36 \times 10^{-8}$ \\
\hline rs73186270 & 7:106444515 & A & G & $\begin{array}{l}\text { intron_variant, } \\
\text { non_coding_transcript_variant }\end{array}$ & $\mathrm{M}$ & RP5-884M6.1 & 0.1 & $-0.885(0.152)$ & $5.74 \times 10^{-9}$ & $-1045(0.242)$ & 0.0000212 & $-0.033(0.006)$ & $5.83 \times 10^{-8}$ \\
\hline rs73186233 & 7:106413337 & A & G & downstream_gene_variant & $\mathrm{M}$ & RP5-884M6.1 & 0.09 & $-0.933(0.161)$ & $5.98 \times 10^{-9}$ & $-1091(0.26)$ & 0.00001618 & $-0.037(0.007)$ & $1.21 \times 10^{-7}$ \\
\hline rs17477708 & 7:106433932 & A & G & downstream_gene_variant & $\mathrm{M}$ & RP5-884M6.1 & 0.09 & $-0.929(0.16)$ & $6.31 \times 10^{-9}$ & $-1106(0.258)$ & 0.00002551 & $-0.036(0.007)$ & $5.57 \times 10^{-8}$ \\
\hline rs73186245 & 7:106422325 & $\mathrm{T}$ & C & $\begin{array}{c}\text { intron_variant, } \\
\text { non_coding_transcript_variant }\end{array}$ & M & RP5-884M6.1 & 0.09 & $-0.933(0.16)$ & $6.37 \times 10^{-9}$ & $-1105(0.258)$ & 0.00001776 & $-0.037(0.007)$ & $8.85 \times 10^{-8}$ \\
\hline rs73186239 & 7:106417063 & A & G & $\begin{array}{c}\text { intron_variant, } \\
\text { non_coding_transcript_variant }\end{array}$ & M & RP5-884M6.1 & 0.09 & $-0.914(0.158)$ & $6.96 \times 10^{-9}$ & $-1069(0.253)$ & 0.00002631 & $-0.036(0.007)$ & $7.21 \times 10^{-8}$ \\
\hline rs73186269 & 7:106441672 & $\mathrm{T}$ & C & intergenic_variant & M & - & 0.09 & $-0.919(0.158)$ & $6.99 \times 10^{-9}$ & $-1081(0.254)$ & 0.00002455 & $-0.036(0.007)$ & $6.81 \times 10^{-8}$ \\
\hline rs75209503 & $7: 106435698$ & $\mathrm{~T}$ & C & intergenic_variant & M & - & 0.09 & $-0.909(0.158)$ & $7.97 \times 10^{-9}$ & $-1067(0.253)$ & 0.00002527 & $-0.036(0.007)$ & $8.06 \times 10^{-8}$ \\
\hline \multicolumn{14}{|l|}{ (b) } \\
\hline SNP & CHR:BP & A1 & A2 & Consequence & IMPACT & Gene & A1freq & $\begin{array}{c}\text { Beta } \\
\text { (SE)-MTAG }\end{array}$ & P-MTAG & Beta (SE)-MPV & P-MPV & $\begin{array}{l}\text { Beta(SE)- } \\
\text { PD_AAO }\end{array}$ & P-PD_AAO \\
\hline rs17477708 & 7:106433932 & A & G & $\begin{array}{l}\text { intron_variant, } \\
\text { non_coding_transcript_variant }\end{array}$ & M & LINC02577 & 0.27 & $-0.022(0.004)$ & $3.26 \times 10^{-8}$ & $-0.215(0.134)$ & $2.55 \times 10^{-5}$ & $-0.022(0.004)$ & $5.57 \times 10^{-8}$ \\
\hline rs73186270 & 7:106444515 & A & G & $\begin{array}{c}\text { intron_variant, } \\
\text { non_coding_transcript_variant }\end{array}$ & M & LINC02577 & 0.4 & $-0.02(0.004)$ & $3.39 \times 10^{-8}$ & $-0.33(0.133)$ & $2.12 \times 10^{-5}$ & $-0.02(0.004)$ & $5.83 \times 10^{-8}$ \\
\hline rs7310366 & 12:123069722 & $\mathrm{T}$ & C & intron_variant & M & KNTC1 & 0.4 & $-0.02(0.004)$ & $3.81 \times 10^{-8}$ & $-0.321(0.133)$ & 0.008285 & $-0.02(0.004)$ & $5.09 \times 10^{-8}$ \\
\hline rs73186269 & $7: 106441672$ & $\mathrm{~T}$ & C & $\begin{array}{c}\text { intron_variant, } \\
\text { non_coding_transcript_variant }\end{array}$ & M & LINC02577 & 0.97 & $0.057(0.01)$ & $3.98 \times 10^{-8}$ & $0.773(0.398)$ & $2.46 \times 10^{-5}$ & $0.056(0.01)$ & $6.81 \times 10^{-8}$ \\
\hline rs13333879 & $16: 526435$ & $\mathrm{~T}$ & $\mathrm{C}$ & intron_variant & $\mathrm{M}$ & RAB11FIP3 & 0.89 & $-0.032(0.006)$ & $4.04 \times 10^{-8}$ & $-0.412(0.209)$ & 0.005233 & $-0.032(0.006)$ & $5.26 \times 10^{-8}$ \\
\hline rs16835687 & 3:124327247 & A & G & intron_variant & M & KALRN & 0.17 & $0.027(0.005)$ & $4.08 \times 10^{-8}$ & $0.483(0.189)$ & 0.01256 & $0.027(0.005)$ & $5.20 \times 10^{-8}$ \\
\hline rs11818135 & 10:126323224 & A & C & intron_variant & M & FAM53B & 0.27 & $-0.022(0.004)$ & $4.17 \times 10^{-8}$ & $-0.288(0.162)$ & 0.01054 & $-0.022(0.004)$ & $5.24 \times 10^{-8}$ \\
\hline rs11065565 & 12:121853164 & $\mathrm{T}$ & $\mathrm{C}$ & intron_variant & M & RNF34 & 0.92 & $-0.037(0.007)$ & $4.22 \times 10^{-8}$ & $-0.71(0.254)$ & 0.01601 & $-0.037(0.007)$ & $5.24 \times 10^{-8}$ \\
\hline rs73186239 & 7:106417063 & A & G & $\begin{array}{c}\text { intron_variant, } \\
\text { non_coding_transcript_variant }\end{array}$ & M & LINC02577 & 0.1 & $0.033(0.006)$ & $4.23 \times 10^{-8}$ & $0.519(0.208)$ & $2.63 \times 10^{-5}$ & $0.032(0.006)$ & $7.21 \times 10^{-8}$ \\
\hline rs3817544 & 12:121882819 & $\mathrm{T}$ & $\mathrm{C}$ & intron_variant & $\mathrm{M}$ & KDM2B & 0.28 & $-0.022(0.004)$ & $4.25 \times 10^{-8}$ & $-0.215(0.131)$ & 0.01663 & $-0.022(0.004)$ & $5.26 \times 10^{-8}$ \\
\hline rs73186248 & $7: 106423109$ & A & G & downstream_gene_variant & $\mathrm{M}$ & LINC02577 & 0.92 & $-0.038(0.007)$ & $4.25 \times 10^{-8}$ & $-1.084(0.258)$ & $1.78 \times 10^{-5}$ & $-0.037(0.007)$ & $7.36 \times 10^{-8}$ \\
\hline rs6761213 & 2:223870120 & A & G & intergenic_variant & M & - & 0.74 & $-0.023(0.004)$ & $4.29 \times 10^{-8}$ & $-0.26(0.146)$ & 0.03182 & $-0.023(0.004)$ & $5.09 \times 10^{-8}$ \\
\hline
\end{tabular}


Table 2. Cont.

\begin{tabular}{|c|c|c|c|c|c|c|c|c|c|c|c|c|c|}
\hline (b) & & & & & & & & & & & & & \\
\hline SNP & CHR:BP & A1 & A2 & Consequence & IMPACT & Gene & A1freq & $\begin{array}{c}\text { Beta } \\
\text { (SE)-MTAG } \\
\end{array}$ & p-MTAG & $\begin{array}{c}\text { Beta } \\
\text { (SE)-PD_AAO } \\
\end{array}$ & P-PD_AAO & Beta(SE)-MPV & P-MPV \\
\hline rs76123955 & 10:64855929 & A & G & intergenic_variant & $\mathrm{M}$ & - & 0.92 & $-0.037(0.007)$ & $4.42 \times 10^{-8}$ & $-0.711(0.255)$ & 0.05053 & $-0.036(0.007)$ & $5.03 \times^{-8}$ \\
\hline rs113899647 & 10:64850074 & $\mathrm{T}$ & C & intergenic_variant & $\mathrm{M}$ & - & 0.12 & $-0.031(0.006)$ & $4.49 \times 10^{-8}$ & $-0.441(0.22)$ & 0.05195 & $-0.031(0.006)$ & $5.09 \times 10^{-8}$ \\
\hline rs74760108 & $6: 25473784$ & A & G & intron_variant & $\mathrm{M}$ & CARMIL1 & 0.42 & $-0.02(0.004)$ & $4.49 \times 10^{-8}$ & $-0.231(0.124)$ & 0.03488 & $-0.02(0.004)$ & $5.35 \times 10^{-8}$ \\
\hline rs35041742 & 17:20036253 & $\mathrm{T}$ & C & intron_variant & $\mathrm{M}$ & SPECC1 & 0.42 & $-0.02(0.004)$ & $4.60 \times 10^{-8}$ & $-0.231(0.124)$ & 0.06307 & $-0.02(0.004)$ & $5.17 \times 10^{-8}$ \\
\hline rs62261974 & 3:107296969 & A & G & intron_variant & $\mathrm{M}$ & BBX & 0.4 & $-0.02(0.004)$ & $4.60 \times 10^{-8}$ & $-0.32(0.133)$ & 0.0677 & $-0.02(0.004)$ & $5.03 \times 10^{-8}$ \\
\hline rs4081775 & 12:121847579 & A & G & intron_variant & $\mathrm{M}$ & RNF34 & 0.58 & $0.02(0.004)$ & $4.64 \times 10^{-8}$ & $0.227(0.124)$ & 0.01254 & $0.02(0.004)$ & $5.86 \times 10^{-8}$ \\
\hline rs1765710 & $1: 45936700$ & $\mathrm{~T}$ & C & intron_variant & $\mathrm{M}$ & TESK2 & 0.7 & $0.022(0.004)$ & $4.66 \times 10^{-8}$ & $0.278(0.143)$ & 0.07405 & $0.022(0.004)$ & $5.14 \times 10^{-8}$ \\
\hline rs11900857 & 2:191659011 & $\mathrm{T}$ & C & $\begin{array}{c}\text { intron_variant, } \\
\text { non_coding_transcript_variant }\end{array}$ & M & - & 0.4 & $-0.02(0.004)$ & $4.66 \times 10^{-8}$ & $-0.333(0.133)$ & 0.07622 & $-0.02(0.004)$ & $5.04 \times 10^{-8}$ \\
\hline rs75209503 & $7: 106435698$ & $\mathrm{~T}$ & C & $\begin{array}{c}\text { intron_variant, } \\
\text { non_coding_transcript_variant }\end{array}$ & $\mathrm{M}$ & LINC02577 & 0.19 & $0.026(0.005)$ & $4.72 \times 10^{-8}$ & $0.334(0.183)$ & $2.53 \times 10^{-5}$ & $0.026(0.005)$ & $8.06 \times 10^{-8}$ \\
\hline rs4003797 & $17: 20288900$ & $\mathrm{~T}$ & C & $\begin{array}{c}\text { intron_variant, } \\
\text { non_coding_transcript_variant }\end{array}$ & M & CCDC144CP & 0.56 & $-0.02(0.004)$ & $4.75 \times 10^{-8}$ & $-0.312(0.141)$ & 0.0674 & $-0.02(0.004)$ & $5.31 \times 10^{-8}$ \\
\hline rs958753 & 2:223995281 & A & C & $\begin{array}{c}\text { intron_variant, } \\
\text { non_coding_transcript_variant }\end{array}$ & M & KCNE4 & 0.19 & $-0.025(0.005)$ & $4.78 \times 10^{-8}$ & $-0.337(0.157)$ & 0.1045 & $-0.025(0.005)$ & $5.09 \times 10^{-8}$ \\
\hline rs34210276 & $5: 77060737$ & $\mathrm{~T}$ & C & intron_variant & M & TBCA & 0.35 & $0.021(0.004)$ & $4.80 \times 10^{-8}$ & $0.329(0.124)$ & 0.04518 & $0.021(0.004)$ & $5.42 \times 10^{-8}$ \\
\hline rs4028 & 3:124261869 & A & G & intron_variant & $\mathrm{M}$ & KALRN & 0.92 & $-0.038(0.007)$ & $4.85 \times 10^{-8}$ & $-1.105(0.258)$ & 0.05091 & $-0.037(0.007)$ & $5.50 \times 10^{-8}$ \\
\hline rs11585488 & 1:45617312 & A & G & intron_variant & $\mathrm{M}$ & ZSWIM5 & 0.92 & $-0.037(0.007)$ & $4.86 \times 10^{-8}$ & $-1.069(0.253)$ & 0.04904 & $-0.036(0.007)$ & $5.56 \times 10^{-8}$ \\
\hline rs28678897 & $16: 527485$ & A & G & intron_variant & $\mathrm{M}$ & RAB11FIP3 & 0.92 & $-0.037(0.007)$ & $4.86 \times 10^{-8}$ & $-1.081(0.254)$ & 0.005302 & $-0.036(0.007)$ & $6.31 \times 10^{-8}$ \\
\hline rs10072700 & 5:131816903 & A & C & downstream_gene_variant & $\mathrm{M}$ & IRF1 & 0.16 & $-0.027(0.005)$ & $4.86 \times 10^{-8}$ & $-0.344(0.163)$ & 0.1091 & $-0.027(0.005)$ & $5.22 \times 10^{-8}$ \\
\hline rs36051592 & 17:20035020 & $\mathrm{T}$ & C & intron_variant & $\mathrm{M}$ & SPECC1 & 0.92 & $-0.037(0.007)$ & $4.87 \times 10^{-8}$ & $-1.067(0.253)$ & 0.0624 & $-0.036(0.007)$ & $5.49 \times 10^{-8}$ \\
\hline rs169632 & 12:121588545 & $\mathrm{T}$ & C & $\begin{array}{c}\text { intron_variant, } \\
\text { NMD_transcript_variant }\end{array}$ & M & $\mathrm{P} 2 \mathrm{RX7}$ & 0.97 & $0.057(0.01)$ & $4.93 \times 10^{-8}$ & $0.775(0.396)$ & 0.1002 & $0.056(0.01)$ & $5.34 \times 10^{-8}$ \\
\hline rs10849880 & 12:121859844 & A & C & intron_variant & $\mathrm{M}$ & RNF34 & 0.23 & $-0.024(0.004)$ & $4.99 \times 10^{-8}$ & $-0.179(0.137)$ & 0.01321 & $-0.024(0.004)$ & $6.28 \times 10^{-8}$ \\
\hline rs79502317 & 11:61736136 & $\mathrm{T}$ & G & upstream_gene_variant & $\mathrm{M}$ & FTH1 & 0.87 & $0.03(0.005)$ & $5.00 \times 10^{-8}$ & $0.302(0.186)$ & 0.191 & $0.029(0.005)$ & $5.06 \times 10^{-8}$ \\
\hline
\end{tabular}

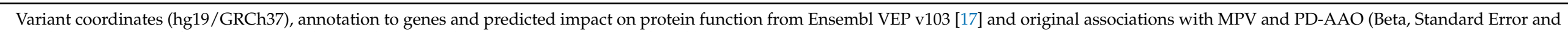
$p$-value) are reported for each variant, along with their multitrait associations as produced by MTAG. 
(a)

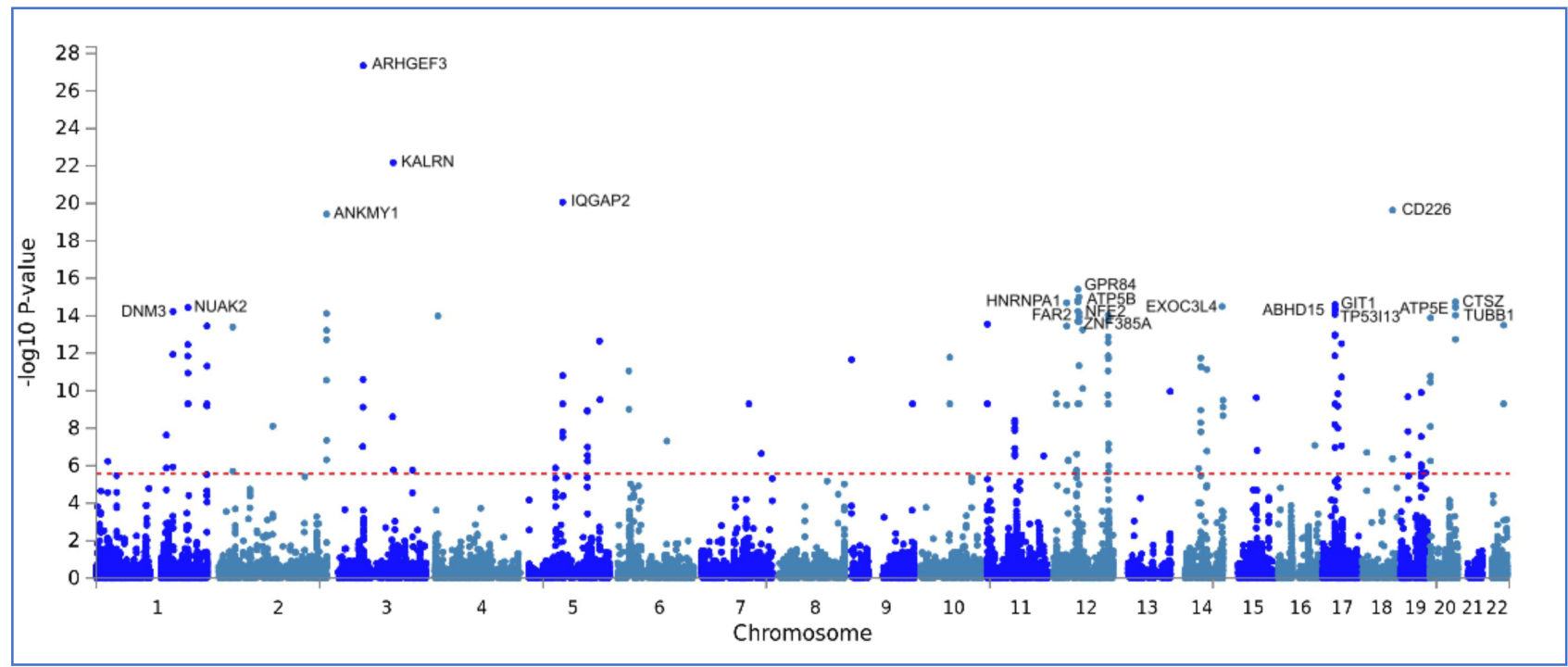

(b)

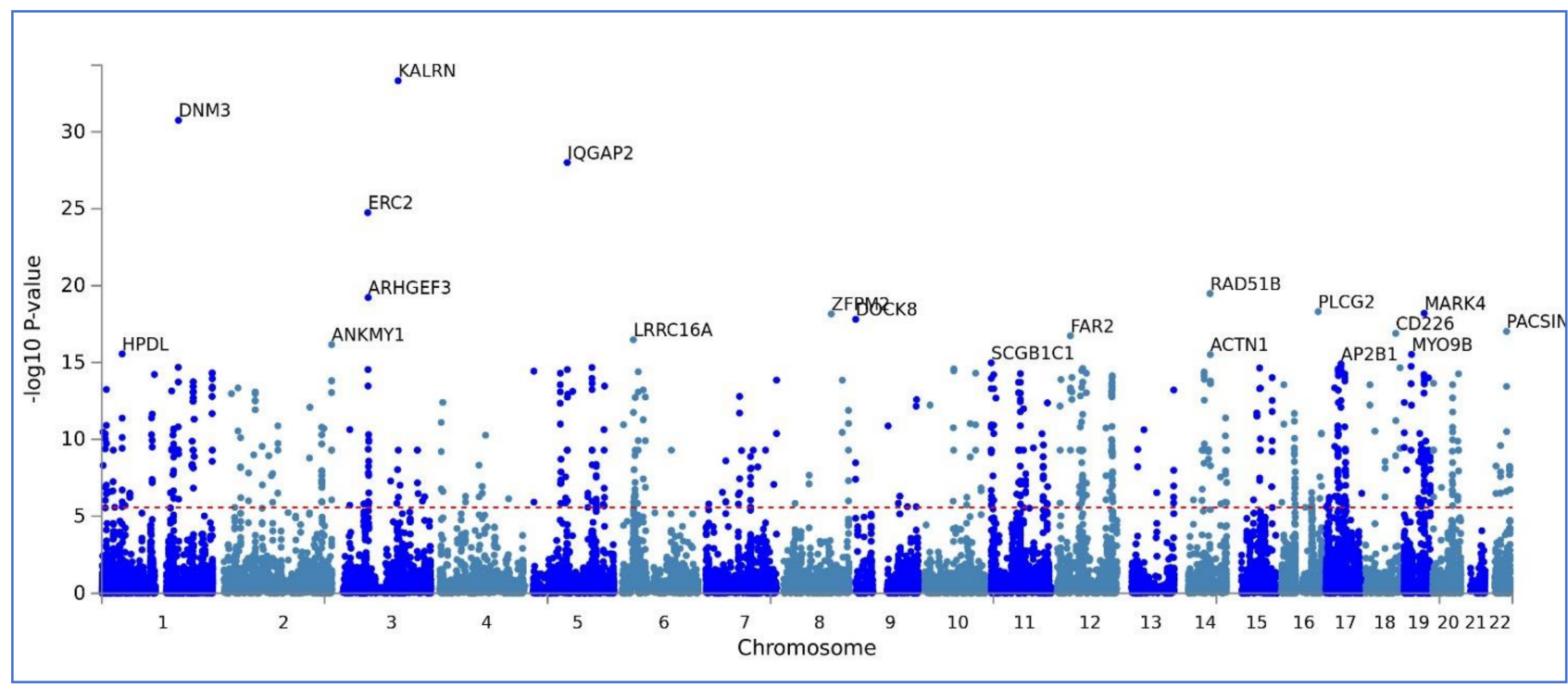

Figure 2. Manhattan Plot of gene-based enrichments of multitrait single-variant associations with (a) PD-AAO and (b) MPV. 
Table 3. Gene-Set enrichments of multitrait single-variant associations with (a) PD-AAO and (b) MPV.

\begin{tabular}{|c|c|c|c|c|c|c|}
\hline \multicolumn{7}{|l|}{ (a) } \\
\hline NGENES & BETA & BETA STD & SE & $p$ & Pbonf & FULL NAME \\
\hline 12 & 2.04 & 0.05 & 0.30 & $5.42 \times 10^{-12}$ & $8.39 \times 10^{-8}$ & $\begin{array}{l}\text { GO: thrombin-activated receptor } \\
\text { signalling pathway }\end{array}$ \\
\hline 323 & 0.35 & 0.05 & 0.05 & $1.68 \times 10^{-11}$ & $2.60 \times 10^{-7}$ & GO: coagulation \\
\hline 591 & 0.23 & 0.04 & 0.04 & $7.28 \times 10^{-10}$ & $1.13 \times 10^{-5}$ & $\begin{array}{l}\text { Curated gene sets: } \\
\text { reactome hemostasis }\end{array}$ \\
\hline 161 & 0.43 & 0.04 & 0.08 & $4.87 \times 10^{-9}$ & $7.55 \times 10^{-5}$ & $\begin{array}{l}\text { Curated gene sets: reactome factors } \\
\text { involved in } \\
\text { megakaryocyte development and } \\
\text { platelet production }\end{array}$ \\
\hline 475 & 0.23 & 0.04 & 0.04 & $5.03 \times 10^{-8}$ & $7.78 \times 10^{-4}$ & GO: regulation of body fluid levels \\
\hline 518 & 0.21 & 0.03 & 0.04 & $5.68 \times 10^{-8}$ & $8.79 \times 10^{-4}$ & GO: wound healing \\
\hline 5 & 2.32 & 0.04 & 0.44 & $7.37 \times 10^{-8}$ & $1.14 \times 10^{-3}$ & $\begin{array}{l}\text { GO: thrombin-activated } \\
\text { receptor activity }\end{array}$ \\
\hline 178 & 0.34 & 0.03 & 0.07 & $1.08 \times 10^{-7}$ & $1.67 \times 10^{-3}$ & $\begin{array}{l}\text { Curated gene sets: wierenga stat5a } \\
\text { targets dn }\end{array}$ \\
\hline 5 & 2.22 & 0.04 & 0.43 & $1.43 \times 10^{-7}$ & $2.21 \times 10^{-3}$ & $\begin{array}{l}\text { GO: protein localization to } \\
\text { juxtaparanode region of axon }\end{array}$ \\
\hline 7 & 2.06 & 0.04 & 0.40 & $1.46 \times 10^{-7}$ & $2.26 \times 10^{-3}$ & $\begin{array}{l}\text { Curated gene sets: reactome } \\
\text { erythropoietin activates } \\
\text { phospholipase c gamma plcg }\end{array}$ \\
\hline 627 & 0.19 & 0.03 & 0.04 & $2.14 \times 10^{-7}$ & $3.31 \times 10^{-3}$ & GO: response to wounding \\
\hline 7 & 2.05 & 0.04 & 0.41 & $3.81 \times 10^{-7}$ & $5.89 \times 10^{-3}$ & $\begin{array}{l}\text { GO: positive regulation of fc } \\
\text { receptor-mediated } \\
\text { stimulatory signalling pathway }\end{array}$ \\
\hline 5 & 2.13 & 0.03 & 0.45 & $1.05 \times 10^{-6}$ & $1.63 \times 10^{-2}$ & $\begin{array}{l}\text { GO: type } 5 \text { metabotropic glutamate } \\
\text { receptor binding }\end{array}$ \\
\hline 9 & 1.70 & 0.04 & 0.37 & $2.73 \times 10^{-6}$ & $4.22 \times 10^{-2}$ & GO: exocyst localization \\
\hline 25 & 0.87 & 0.03 & 0.19 & $2.86 \times 10^{-6}$ & $4.43 \times 10^{-2}$ & $\begin{array}{l}\text { GO: cellular protein-containing } \\
\text { complex } \times \text { localization }\end{array}$ \\
\hline 18 & 0.99 & 0.03 & 0.22 & $2.91 \times 10^{-6}$ & $4.50 \times 10^{-2}$ & GO: platelet morphogenesis \\
\hline \multicolumn{7}{|l|}{ (b) } \\
\hline NGENES & BETA & BETA STD & SE & $p$ & Pbonf & FULL NAME \\
\hline 323 & 0.42 & 0.05 & 0.07 & $1.86 \times 10^{-9}$ & $2.87 \times 10^{-5}$ & GO: coagulation \\
\hline 161 & 0.60 & 0.06 & 0.10 & $2.82 \times 10^{-9}$ & $4.37 \times 10^{-5}$ & $\begin{array}{l}\text { Curated gene sets: reactome factors } \\
\text { involved in megakaryocyte } \\
\text { development and platelet production }\end{array}$ \\
\hline 158 & 0.57 & 0.05 & 0.10 & $4.08 \times 10^{-9}$ & $6.32 \times 10^{-5}$ & GO: rho gtpase binding \\
\hline 591 & 0.29 & 0.05 & 0.05 & $5.47 \times 10^{-9}$ & $8.46 \times 10^{-5}$ & $\begin{array}{l}\text { Curated gene sets: } \\
\text { reactome hemostasis }\end{array}$ \\
\hline 496 & 0.31 & 0.05 & 0.06 & $1.45 \times 10^{-8}$ & $2.25 \times 10^{-4}$ & GO: gtpase binding \\
\hline 438 & 0.34 & 0.05 & 0.06 & $1.72 \times 10^{-8}$ & $2.66 \times 10^{-4}$ & GO: regulation of gtpase activity \\
\hline 403 & 0.33 & 0.05 & 0.06 & $3.92 \times 10^{-8}$ & $6.07 \times 10^{-4}$ & GO: small gtpase binding \\
\hline 371 & 0.34 & 0.05 & 0.07 & $1.04 \times 10^{-7}$ & $1.61 \times 10^{-3}$ & $\begin{array}{l}\text { GO: positive regulation of } \\
\text { gtpase activity }\end{array}$ \\
\hline
\end{tabular}


Table 3. Cont.

\begin{tabular}{ccccccc}
\hline \multicolumn{1}{l}{ N) } & BETA & BETA STD & SE & $p$ & Pbonf & FULL NAME \\
\hline 7 & 2.77 & 0.05 & 0.55 & $2.13 \times 10^{-7}$ & $3.30 \times 10^{-3}$ & $\begin{array}{c}\text { Curated gene sets: reactome } \\
\text { erythropoietin activates } \\
\text { phospholipase c gamma plcg }\end{array}$ \\
\hline 178 & 0.45 & 0.04 & 0.09 & $3.15 \times 10^{-7}$ & $4.88 \times 10^{-3}$ & $\begin{array}{c}\text { Curated gene sets: wierenga stat5a } \\
\text { targets dn }\end{array}$ \\
\hline 59 & 0.83 & 0.05 & 0.17 & $3.70 \times 10^{-7}$ & $5.72 \times 10^{-3}$ & GO: rac gtpase binding \\
\hline 275 & 0.36 & 0.04 & 0.08 & $9.67 \times 10^{-7}$ & $1.50 \times 10^{-2}$ & GO: gtpase regulator activity \\
\hline 475 & 0.28 & 0.04 & 0.06 & $1.03 \times 10^{-6}$ & $1.60 \times 10^{-2}$ & GO: regulation of body fluid levels \\
\hline
\end{tabular}

The dashed line represents the Bonferroni-adjusted significance threshold for the gene-enrichment test $\left(\alpha=2.6 \times 10^{-6}\right)$. Only the 20 most enriched genes for each analysis are labeled (see Table S1a,b for a full list).

\section{Discussion}

In this work, we carried out a detailed analysis of the genomic overlap between platelet parameters' variability and age at onset of Parkinson Disease, first by investigating the presence and entity of the overlap and then by attempting to identify single variants, genes and pathways at the basis of this co-heritability. We observed a significant negative genetic correlation between PD-AAO and MPV, suggesting that there may be a link between platelet parameters and an important endophenotype of PD. However, this relation is in contrast with a study reporting higher MPV in PD cases compared to controls [3] and with previous reports associating lower MPV with increased PD severity [3,4]. Indeed, lower AAO is usually associated with an increased H\&Y score as the disease progresses, since neurodegeneration is expected to have more time to exert disruptive effects in younger rather than older PD patients. Of note, lower MPV is also associated with an increased platelet activity, a characteristic which was found in PD cases compared to controls [18], and it is indicative of increased circulating inflammation levels [19]. These apparently contrasting findings may be explained by the existence of a PD (sporadic and late onset) subtype which might be more influenced by systemic inflammation, be it of environmental or of genetic origins, in line with previous evidence $[18,20]$. While research on PD etiology has not yet reached such a fine-grained resolution, evidence that serum levels of inflammatory cytokines correlate with H\&Y staging [21] may support this hypothesis, which warrants further epidemiological and functional studies in order to be verified.

The multitrait analysis of PD-AAO and MPV revealed diverse novel variants associated with both traits, which were not detected in the original GWAS studies $[8,16]$. Among these variants, significant associations were detected in genes previously implicated in PD pathology or coagulation pathways, like KALRN, DNM3, ARHGEF3 and IQGAP2.

A gene-based enrichment test of multitrait associations revealed different overlaps between PD-AAO and MPV. Indeed, the most significantly enriched genes for associations with PD-AAO-ARHGEF3, KALRN and IQGAP2 - were also among those most enriched for associations with MPV. Interestingly, some of these genes have been implicated in known neurodegenerative related processes and diseases, like depression (ARHGEF3) [22], Alzheimer's Disease and schizophrenia (KALRN) [23], or even Parkinson Disease (KALRN, IQGAP2) [23,24]. Of particular interest, KALRN encodes the kalirin protein, a key regulator of structural and functional plasticity at dendritic spines [25], which interacts with synphilin-1 (leading to the recruitment of aggresomes) and, indirectly, with alfa-synuclein, a key protein in PD etiology [26].

A pathway-based enrichment analysis further supported the idea of a common genetic and biological basis for PD-AAO and MPV, since both traits showed an enrichment of 
coagulation-related pathways. While this may result as being obvious for MPV, it may not appear that obvious for PD-AAO, especially considering that PD is, among all common neurodegenerative disorders, the least investigated for associations with platelet parameters (see the review by Izzi et al., 2020 [27]). In particular, pathways like coagulation, megakaryocyte development and platelet production may be directly involved in this common link. It is known that PD is accompanied by dysregulation in blood clotting, as previously suggested by proteome analyses within two mouse models of PD [28]. Our results support the hypothesis that the abovementioned pathways may be simultaneously related to both platelet parameters' variability and to neuropathological mechanisms in sporadic, lateonset forms of PD. Further functional studies are warranted to investigate this fascinating hypothesis, as has been successfully done for Alzheimer's disease [29]. Indeed, these candidate pathways may be functionally investigated at more fine-grained resolutions within both cellular and animal models of PD, with the latter being fundamental to build a link between platelet parameters' variability and the disease.

\section{Strengths and Limitations}

While this study provides novel insights on the genetic overlap between Parkinson Disease and platelets, it also presents some limitations. First, the analysis is exploratory in nature, providing useful indications on the genes and pathways potentially involved in the genomic overlap, but it is not indicative of a potential for MPV to be a predictive biomarker of PD age at onset. Observational longitudinal analysis in population cohorts, large clinical studies and functional studies in animal models are warranted to clarify this aspect. Moreover, this study is only based on common variants and does not consider rare variants, which have a major influence on early-onset/familial PD cases. While in genomic studies based on huge sample sizes (in the order of tens or hundreds of thousands) and on a high number of variants (in the order of millions) significant $p$-values are often associated with very small effect sizes, the use of publicly available GWAS summary statistics favors notable advances in gaining knowledge on unexplored genomic links. Still, it is worth underlining again that statistical findings need to be validated at the functional level, in vivo and in vitro.

Overall, this study represents a first attempt to clarify the genetic scenario underlying the shared biology between platelets and Parkinson Disease, and a potential starting point for functional studies in animal models.

Supplementary Materials: The following are available online at https:/ / www.mdpi.com/article/10 .3390/app11156927/s1, Table S1a. Gene-based enrichments of multi-trait associations with PD-AAO, as performed in MAGMA v 1.08. Table S1b. Gene-based enrichments of multi-trait associations with MPV, as performed in MAGMA v 1.08. Table S1c. Gene-set enrichments of multi-trait associations with PD-AAO, as performed in MAGMA v 1.08. Table S1d. Gene-set enrichments of multi-trait associations with MPV, as performed in MAGMA v 1.08.

Author Contributions: G.d.G., C.C. and M.B.D. inspired this research and provided the theoretical background on platelet parameters and potential neurological implications. L.I., G.d.G., A.T. and A.G. conceived the study and designed the analyses. A.T., A.G. and R.P. performed statistical analyses. R.P., M.B.D. and C.C. contributed to the interpretation of genes and pathways identified. A.T., A.G., G.d.G. and L.I. wrote the first draft of the manuscript, and R.P. contributed to its revised form, along with all co-authors. All authors have read and agreed to the published version of the manuscript.

Funding: A.G. was supported by Fondazione Umberto Veronesi. This study was partially supported by the Italian Ministry of Health (Ricerca Corrente 2019-2023).

Institutional Review Board Statement: Not applicable.

Informed Consent Statement: Not applicable.

Data Availability Statement: Data available in a publicly accessible repository that does not issue DOIs. Publicly available datasets were analyzed in this study. These data can be found here: $[5,8]$.

Conflicts of Interest: The authors declare no competing financial interests. 


\section{References}

1. Tirozzi, A.; Izzi, B.; Noro, F.; Marotta, A.; Gianfagna, F.; Hoylaerts, M.F.; Cerletti, C.; Donati, M.B.; De Gaetano, G.; Iacoviello, L.; et al. Assessing genetic overlap between platelet parameters and neurodegenerative disorders. Front. Immunol. 2020, 11, 02127. [CrossRef] [PubMed]

2. Canobbio, I. Blood platelets: Circulating mirrors of neurons? Res. Pract. Thromb. Haemost. 2019, 3, 564-565. [CrossRef]

3. Geyik, S.; Yigiter, R.; Akgul, G.P.; Elci, M.; Firat, Y.E. The Relationship between Parkinson's Disease and Mean Platelet Volume. Park. Hast. Hareket Bozuklukları Derg. 2016, 19, 31-34. [CrossRef]

4. Koçer, A.; Yaman, A.; Niftaliyev, E.; Dürüyen, H.; Eryılmaz, M.; Koçer, E. Assessment of platelet indices in patients with neurodegenerative diseases: Mean platelet volume was increased in patients with Parkinson's disease. Curr. Gerontol. Geriatr. Res. 2013, 2013, 986254. [CrossRef]

5. Nalls, M.A.; Blauwendraat, C.; Vallerga, C.L.; Heilbron, K.; Bandres-Ciga, S.; Chang, D.; Tan, M.; Kia, D.A.; Noyce, A.J.; Xue, A.; et al. Identification of novel risk loci, causal insights, and heritable risk for Parkinson's disease: A meta-analysis of ge-nome-wide association studies. Lancet Neurol. 2019, 18, 1091-1102. [CrossRef]

6. Bulik-Sullivan, B.K.; Loh, P.R.; Finucane, H.K.; Ripke, S.; Yang, J.; Patterson, N.; Daly, M.J.; Price, A.L.; Neale, B.M. LD Score regression distinguishes confounding from polygenicity in genome-wide association studies. Nat. Genet. 2015, 47, 291-295. [CrossRef]

7. Bulik-Sullivan, B.; Finucane, H.K.; Anttila, V.; Gusev, A.; Day, F.R.; Loh, P.-R.; Duncan, L.E.; Perry, J.R.; Patterson, N.; Robinson, E.; et al. An atlas of genetic correlations across human diseases and traits. Nat. Genet. 2015, 47, 1236-1241. [CrossRef]

8. Astle, W.; Elding, H.; Jiang, T.; Allen, D.; Ruklisa, D.; Mann, A.; Mead, D.; Bouman, H.; Riveros-Mckay, F.; Kostadima, M.A.; et al . The allelic landscape of human blood cell trait variation and links to common complex disease. Cell 2016, 167, 1415-1429.e19. [CrossRef]

9. Gialluisi, A.; Reccia, M.G.; Tirozzi, A.; Nutile, T.; Lombardi, A.; De Sanctis, C.; Varanese, S.; Pietracupa, S.; Modugno, N.; Simeone, A.; et al. Whole exome sequencing study of parkinson disease and related endophenotypes in the Italian population. Front. Neurol. 2020, 10, 1362. [CrossRef]

10. Hapmap3. Available online: https:/ / www.sanger.ac.uk/resources/downloads/human/hapmap3.html (accessed on 23 July 2021).

11. LDSCORE. Available online: https:/ / data.broadinstitute.org/alkesgroup/LDSCORE/w_hm3.snplist (accessed on 23 July 2021).

12. MTAG. Available online: https://github.com/omeed-maghzian/mtag (accessed on 23 July 2021).

13. Turley, P.; Walters, R.K.; Maghzian, O.; Okbay, A.; Lee, J.J.; Fontana, M.A.; Nguyen-Viet, T.A.; Wedow, R.; Zacher, M.; Furlotte, N.A.; et al. Multi-trait analysis of genome-wide association summary statistics using MTAG. Nat. Genet. 2018, 50, 229-237. [CrossRef]

14. De Leeuw, C.A.; Mooij, J.M.; Heskes, T.; Posthuma, D. MAGMA: Generalized gene-set analysis of GWAS data. PLoS Comput. Biol. 2015, 11, e1004219. [CrossRef]

15. Watanabe, K.; Taskesen, E.; Van Bochoven, A.; Posthuma, D. Functional mapping and annotation of genetic associations with FUMA. Nat. Commun. 2017, 8, 1826. [CrossRef]

16. Blauwendraat, C.; Heilbron, K.; Vallerga, C.L.; Bandres-Ciga, S.; Von Coelln, R.; Pihlstrøm, L.; Simón-Sánchez, J.; Schulte, C.; Sharma, M.; Krohn, L.; et al. Parkinson's disease age at onset genome-wide association study: Defining heritability, genetic loci, and $\alpha$-synuclein mechanisms. Mov. Disord. 2019, 34, 866-875. [CrossRef] [PubMed]

17. Ensembl. Available online: https://grch37.ensembl.org/info/docs/tools/vep/index.html (accessed on 23 July 2021).

18. Adams, B.; Nunes, J.M.; Page, M.; Roberts, T.; Carr, J.; Nell, T.; Kell, D.; Pretorius, E. Parkinson's Disease: A systemic inflammatory disease accompanied by bacterial inflammagens. Front. Aging Neurosci. 2019, 11, 210. [CrossRef] [PubMed]

19. Korniluk, A.; Koper-Lenkiewicz, O.M.; Kamińska, J.; Kemona, H.; Dymicka-Piekarska, V. Mean Platelet Volume (MPV): New perspectives for an old marker in the course and prognosis of inflammatory conditions. Mediat. Inflamm. 2019, $2019,1-14$. [CrossRef]

20. Ferrari, C.C.; Tarelli, R. Parkinson's disease and systemic inflammation. Park. Dis. 2011, 2011, 436813. [CrossRef] [PubMed]

21. Csencsits-Smith, K.; Suescun, J.; Li, K.; Luo, S.; Bick, D.L.; Schiess, M. Serum lymphocyte-associated cytokine concentrations change more rapidly over time in multiple system atrophy compared to Parkinson Disease. Neuroimmunomodulation 2016, 23, 301-308. [CrossRef]

22. Jovanova, O.S.; Nedeljkovic, I.; Spieler, D.; Walker, R.M.; Liu, C.; Luciano, M.; Bressler, J.; Brody, J.; Drake, A.J.; Evans, K.L.; et al. DNA Methylation signatures of depressive symptoms in middle-aged and elderly persons: Meta-analysis of multiethnic epigenome-wide studies. JAMA Psychiatry 2018, 75, 949-959. [CrossRef]

23. Parnell, E.; Shapiro, L.P.; Voorn, R.A.; Forrest, M.P.; Jalloul, H.A.; Loizzo, D.D.; Penzes, P. KALRN: A central regulator of synaptic function and synaptopathies. Gene 2021, 768, 145306. [CrossRef]

24. Gialluisi, A.; Izzi, B.; Di Castelnuovo, A.; Cerletti, C.; Donati, M.B.; de Gaetano, G.; Iacoviello, L. Revisiting the link between platelets and depression through genetic epidemiology: New insights from platelet distribution width. Haematologica 2020, 105, e246-e248. [CrossRef]

25. Penzes, P.; Remmers, C. Kalirin signaling: Implications for synaptic pathology. Mol. Neurobiol. 2011, 45, 109-118. [CrossRef] [PubMed]

26. Tsai, Y.-C.; Riess, O.; Soehn, A.S.; Nguyen, H.P. The Guanine Nucleotide exchange factor Kalirin-7 is a novel Synphilin-1 interacting protein and modifies Synphilin-1 aggregate transport and formation. PLoS ONE 2012, 7, e51999. [CrossRef] 
27. Izzi, B.; Tirozzi, A.; Cerletti, C.; Donati, M.B.; de Gaetano, G.; Hoylaerts, M.F.; Iacoviello, L.; Gialluisi, A. Beyond Haemostasis and Thrombosis: Platelets in Depression and Its Co-Morbidities. Int. J. Mol. Sci. 2020, 21, 8817. [CrossRef] [PubMed]

28. Gialluisi, A.; Izzi, B.; Di Castelnuovo, A.; Cerletti, C.; Donati, M.B.; de Gaetano, G.; Iacoviello, L. Lipopolysaccharide-binding protein (LBP) can reverse the amyloid state of fibrin seen or induced in Parkinson's disease. PLoS ONE 2018, 13 , e0192121.

29. Merlini, M.; Rafalski, V.A.; Coronado, P.E.R.; Gill, T.M.; Ellisman, M.; Muthukumar, G.; Subramanian, K.S.; Ryu, J.K.; Syme, C.A.; Davalos, D.; et al. Fibrinogen induces microglia-mediated spine elimination and cognitive impairment in an Alzheimer's disease model. Neuron 2019, 101, 1099-1108.e6. [CrossRef] [PubMed] 